\title{
PTEN signaling is required for the maintenance of spermatogonial stem cells in mouse, by regulating the expressions of PLZF and UTF1
}

Wei Zhou ${ }^{1,2+}$, Hongfang Shao $^{3 \dagger}$, Di Zhang ${ }^{2,4 \dagger}$, Jian Dong ${ }^{2}$, Wei Cheng ${ }^{5}$, Lu Wang ${ }^{2}$, Yincheng Teng ${ }^{3 *}$ and Zhuo $\mathrm{Yu}^{2^{*}}$

\begin{abstract}
Background: Pten plays a crucial role in the stem cell maintenance in a few organs. Pten defect also causes the premature oocytes and ovary aging. We and other groups have found that the phosphatidylinositol-3-OH kinase (PI3K)-Akt signaling regulates the proliferation and differentiation of spermatogonial stem cells (SSCS). PTEN functions as a negative regulator of the PI3K pathway. Thus, we thought that the fate of SSCs might be controlled by Pten.

Results: We report that promyelocytic leukaemia zinc finger (PLZF) and undifferentiated embryonic cell transcription factor 1 (UTF1), both of which are germ cell-specific transcriptional factors, are regulated by Pten. Conditional deletion of Pten leads to reduction in PLZF expression but induction of UTF1, which is associated with SSCs depletion and infertility in males with age.

Conclusion: Our data demonstrate that Pten is required for the long-term maintenance of SSCs and precise regulation of spermatogenesis in mouse. The finding of a Pten-regulated GFR $\alpha 1^{+} / \mathrm{PLZF}^{-} / \mathrm{UTF}^{+}{ }^{+}$progenitor population provides a new insight into the precise mechanisms controlling SSC fate.
\end{abstract}

Keywords: Pten knockout, PLZF, UTF1, Spermatogonial stem cells, PI3K-Akt signaling

\section{Background}

Stem cells are capable of renewing themselves to maintain a stem cell pool as a preserved cell source for tissue homeostasis, while they can also differentiate into mature cells to carry out the function of a specific tissue. The precise balance of self-renewal and differentiation of stem cells is critical for the maintenance and function of a tissue or organ throughout life-time. Similar to other stem cells, spermatogonial stem cells (SSCs) renew themselves

\footnotetext{
*Correspondence: teng_yc@126.com; yuzhuo78@aliyun.com tWei Zhou, Hongfang Shaoa and Di Zhang contributed equally to this work

2 Key Laboratory of Cell Differentiation and Apoptosis of Chinese Ministry of Education, Shanghai Jiao Tong University School of Medicine, Shanghai 200025, China

${ }^{3}$ Centre for Reproductive Medicine, Shanghai Jiao Tong University Affiliated Sixth People Hospital, Shanghai 200233, China Full list of author information is available at the end of the article
}

and meanwhile undergo a dramatic differentiation process-spermatogenesis to generate a large number of sperms consistently. Prior to spermatogenesis, multiple mitotic divisions of SSCs produce subpopulations of SSCs, and the balance of the SSC subpopulations is critical for long-term sperm production. Multiple proteins, such as promyelocytic leukaemia zinc finger (PLZF), GDNF family receptor alpha-1 (GFR $\alpha 1$ ) and undifferentiated embryonic cell transcription factor 1 (UTF1), are expressed in SSC subpopulations, which plays a crucial role in the maintenance of SSC pool. PLZF and GFR $\alpha 1$ are required in germ cells for stem cell self-renewal [1-3], whereas UTF1 is restricted to a small subset of spermatogonia that make the cells maintain the ability of differentiation $[4,5]$.

PTEN signaling is critical in governing the stem cell pool not only in the blood system and central neural 
system but also in reproductive system [6-8]. The loss of Pten in ovary via conditional knockout triggers premature of oocytes and ovary aging [8]. On the other hand, we and other groups have revealed that the phosphatidylinositol3-OH kinase(PI3K)/Akt/S6 pathway is a critical signaling in controlling the proliferation and division of SSCs. Disruption of this signaling or Akt knockout leads to the loss of spermatogonial cells and infertility in males $[9,10]$. PTEN is a major negative regulator of PI3K signaling [11, 12]. To understand the function of Pten in regulating SSC fate and fertility in male mouse, we generated conditional Pten knockout males using germ cell specific Cre strain, the Stra8-Cre mouse. It was turned out that the loss of Pten caused reduction of PLZF expression, but induction of UTF1. Thus, conditional Pten knockout leads to depletion of SSC pool and infertility with age.

\section{Results}

\section{Conditional deletion of Pten in spermatogonial cells in mice}

STRA8 is a germ-cell-specific protein and is expressed through neonatal spermatogonial cells to meiotic cells [13]. In the Stra8-EGFP transgenic mice, spermatogonial stem cells can be labeled by EGFP as characterized by transplantation assay [14]. Therefore, Stra8-Cre can be applied to generate SSC-specific gene knockout model. We created SSC-Pten null mice by crossing Pten ${ }^{\text {LoxP/LoxP }}$ mice with Stra8-Cre mice. The Pten knockout genotype was identified by examining the genomic allele of Pten (Fig. 1a), the Pten expression in testis sections of 7 dayold males (Fig. 1b) as well as in whole proteins from adult testes (Fig. 1c). Since Pten is also expressed in non-germ cells, we obtained purified haploid spermatids through cell sorting from adult mice to confirm the absence of Pten expression in germ cells from crossed mice (Fig. 1d).

\section{Conditional deletion of Pten caused overgrowth of testes followed by shrinking and sterility with age}

The males with Pten-deleted in SSCs were grossly normal through all ages except that the size of testes was of overgrowth within the first 50 days after birth then shrunk afterwards (Fig. 2a). Fertility was found lower in the young Pten-deleted males and it was lost progressively after 60 days as measured by mating with wild type females (Fig. 2b). The morphology of testicular tubules cross-section was abnormal as showing larger lumen and
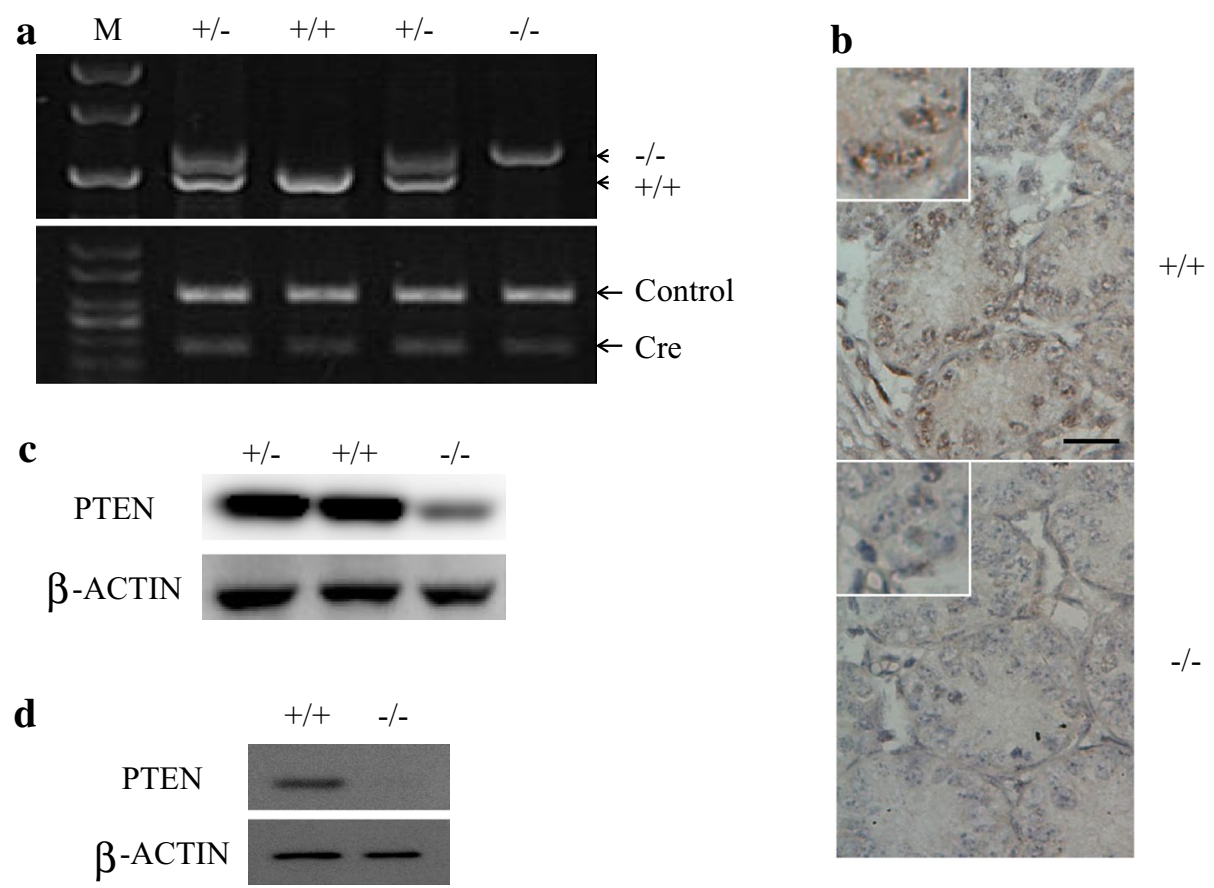

Fig. 1 Cre-recombinase mediated deletion of Pten in SSCs. a PCR analysis of genotype of Pten knockout (KO) mice. Short arrows denote the predicted size of Pten KO and wild-type (WT) alleles, long arrows denote the predicted size of Cre and internal positive control (+/+, wild type; +/-, heterozygote; -/-,homozygote; M, Marker). b Immunostaining of PTEN in cross sections of 7 day-old testis. The cytoplasmic staining was observed in spermatogonial cells from Pten ${ }^{+/+}$section, whereas it was absent in the Pten ${ }^{-/-}$section (scale bar is $20 \mu \mathrm{m}$ ). c Western blot analysis of PTEN expression in whole testis of 32 day-old Pten ${ }^{+/+}$, Pten $^{+/-}$, and Pten ${ }^{-/-}$mice. PTEN is also expressed in nongerminal cells, so PTEN expression was detected in Pten ${ }^{-/-}$testes, but was significantly lower compared with Pten ${ }^{+/+}$and Pten ${ }^{+/-}$testes. $\mathbf{d}$ Absence of PTEN expression in haploid cells from $P$ ten $^{-/-}$testes. Haploid sperms were isolated from 79 day-old testes of Pten ${ }^{+/+}$and Pten ${ }^{-/-}$mice separately by sorting using flow cytometer, then were subjected to Western blotting analysis. 


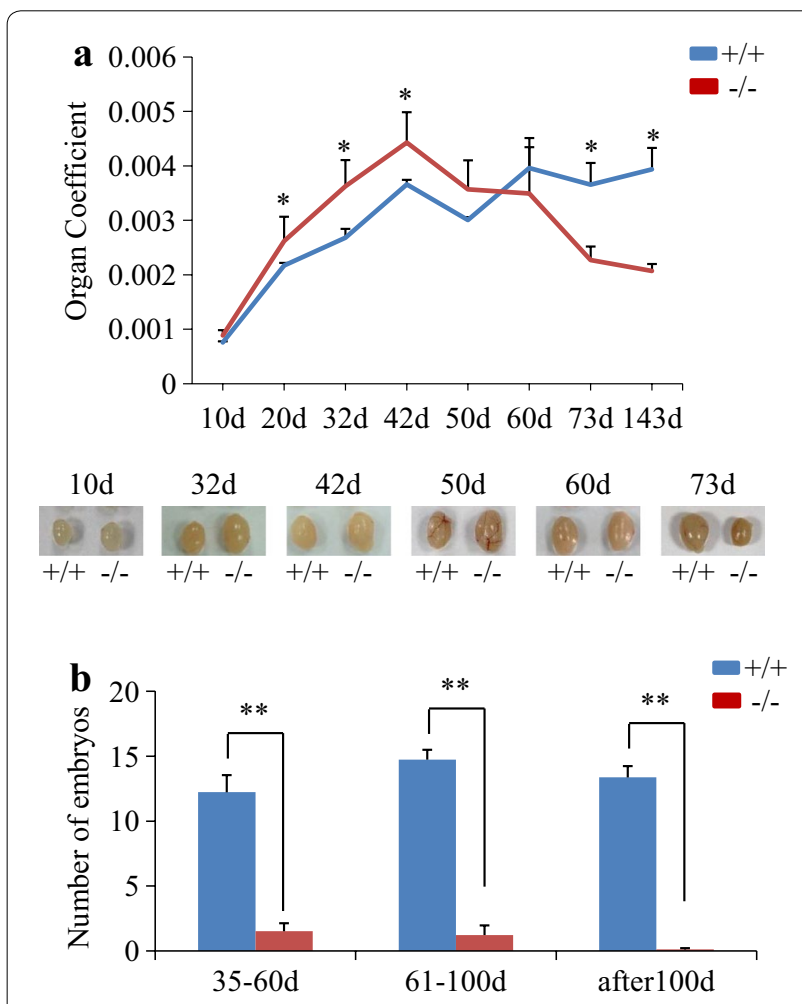

Fig. 2 Overgrowth of testes and fertility loss in Pten ${ }^{-/-}$mice. a Curve of organ coefficient of Pten ${ }^{+/+}$and $P$ ten $^{-/-}$testes with age. Horizontal bars indicate mean values, $n=3{ }^{*} P<0.05$. $\mathbf{b}$ In vivo fertility assay of Pten ${ }^{+/+}$and Pten ${ }^{-/-}$mice. The mating test was divided into three groups according to the male's age: 35-60 days old, 61-100 days old, and over 100 days old. Horizontal bars indicate mean values, $n=22$ ** $P<0.01$.

failure of spermatogenesis with age (Fig. 3a). Furthermore, the rate of spermatogenesis was found very low in the Pten-deleted males during young ages, and severe loss of mature sperm production in the adult males epididymis after age of 60 days (Fig. $3 \mathrm{~b}$ ). This overall phenotype is very similar to that of Pten knockout in bone marrow stem cells and central neural stem cells as overexpansion of short-term hemeatopoietic stem cell pool and enlarged brain [6-8]. Possibly, the loss of Pten might disturb the balance of self-renewal and differentiation and promote excessive differentiation-associated proliferation of SSCs, thereby giving rise to enlarged testes in young mice but causing the depletion of stem cell source and infertility with age.

\section{Loss of Pten led to reduction of SSCs in neonatal males}

To examine whether the self-renewal of SSCs was influenced by the absence of Pten, we used testis cross sections from 7 day-old pups and calculated the number of SSCs. The amount of SSCs expressing both GFR $\alpha 1$ and PLZF was reduced significantly (Fig. 4a, e, f) indicating that Pten played a crucial role in SSC self-renewal in the neonatal testis. To further reveal the molecular mechanisms accounting for the phenotype of this Pten-knockout testis, we used whole transcriptome sequencing to compare the gene expression profiles between wild type and Pten-knockout testes at 32 days old. Interestingly, we found that the expression of UTF1 significantly increased in the Pten-knockout testes (Additional file 1: Figure $\mathrm{S} 1 \mathrm{~B})$. Next, immunostaining of the cross sections confirmed the increase of UTF1-expressing but PLZF-negative cells in Pten ${ }^{-1-} 7$ day-old testis (Fig. 4b, g). At the age of day 10, similar to that of day 7, SSCs expressing GFR $\alpha 1$ or PLZF decreased (Fig. 4c, h, i). However, the number of $\mathrm{UTF}^{+} / \mathrm{PLZF}^{-}$cells was significantly higher in the Ptenknockout testes (Fig. 4d, j). As a chromatin-associated protein, UTF1 is involved in the initiation of ES cell differentiation [15]. In the testis, it has been evidenced that UTF1 is expressed in a subset of spermatogonial cells and germ cell neoplasms, making the SSCs maintain the ability of differentiation $[4,16]$. In $\mathrm{Pten}^{-1-}$ mouse, we have seldom observed neoplasms in the testes. A number of $\mathrm{UTF}^{+} / \mathrm{PLZF}^{-}$cells and few $\mathrm{UTF}^{-} / \mathrm{PLZF}^{+}$cells were observed in the cross sections of 32 day-old Pten ${ }^{-/-}$ mouse testis by immunostaining (Additional file 1: Figure S1A), which implied that Pten-deletion-induced UTF1 expression might boost SSCs differentiation resulting in testes overgrowth in Pten knockout males.

\section{The PTEN signaling regulated the expression of PLZF and UTF1 in SSCs}

We further examined the expression of GFR $\alpha 1$, PLZF and UTF1 in Pten-deleted testes at day 7 or day 10 using Western blot analysis. Consistent with the data from cross section immunostaining, the expression of GFR $\alpha 1$ and PLZF was reduced at both day 7 and day 10, while UTF1 expression was highly increased at day 10 (Fig. 5a). As the PI3K/AKT/mTOR pathway is an important signaling pathway in regulating the cellular functions, PTEN is a major negative regulator of PI3K signaling. Then, we isolated SSCs from 7 days old pups and cultured them in the presence or absence of PI3K inhibitor or mTOR inhibitor-rapamycin to detect the role of PTEN signaling in SSCs in vitro. We found that PLZF expression was induced by inhibiting the PI3K signaling (Fig. 5b, c). However, in the same cell pool, UTF1 expression was reduced in the presence of $\mathrm{PI} 3 \mathrm{~K}$ inhibitor or rapamycin (Fig. 5b, c), implying the presence of a reversal relationship between PLZF and UTF1 in the context of the PTEN/PI3K signaling. Furthermore, UTF1 expression was slightly higher with Rapamycin than with PI3K inhibitor, which indicated that other branched pathways, in addition to mTOR signaling, may participate in this regulation. As we all know, PTEN is an inhibitor of PI3K 


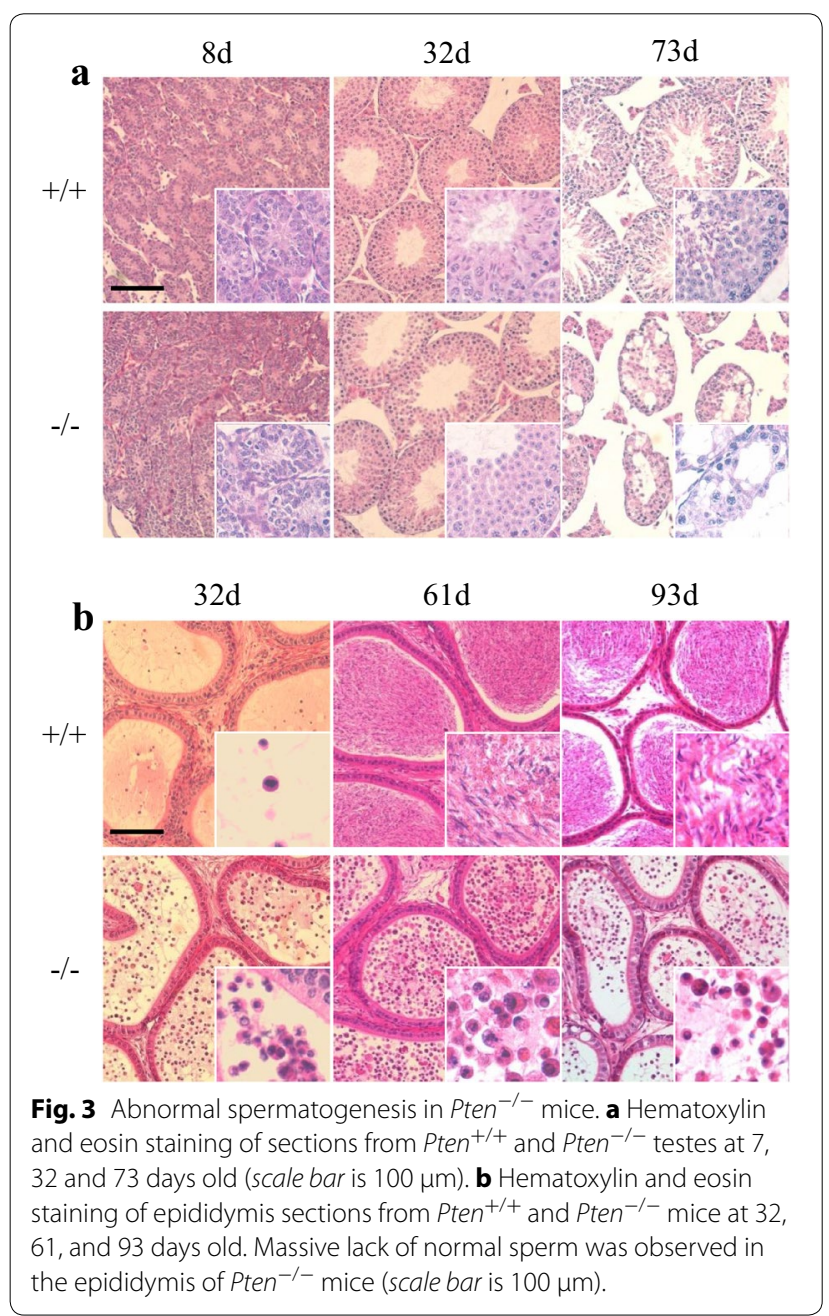

signaling. Thus, we proposed that the PLZF and UTF1 expression was regulated by Pten in SSCs and Pten deletion led to reduction in PLZF expression associated with the increase of UTF1 expression.

\section{A putative model of SSC subpopulation fate controlled by Pten}

To further identify the properties of UTF1 $1^{+}, \mathrm{PLZF}^{+}$and GFR $\alpha 1^{+}$cells, we performed whole mount staining in 7 day-old tubules to locate their expression in SSC population. As shown in Fig. 6a, although most UTF1 positive cells are positive to both GFR $\alpha 1$ and PLZF, a subpopulation of UTF $1^{+} / \mathrm{GFR} \alpha 1^{+} / \mathrm{PLZF}^{-}$SSCs is present. UTF1 protein, which is revealed as a chromatin-associated protein [15], loads on chromosomes in GFR $\alpha 1^{+} / \mathrm{PLZF}^{-}$SSCs (Fig. 6a, upper panel). We observed the dividing spermatogonial cells expressing UTF1 but neither GFR $\alpha 1$ nor PLZF in the 7 day-old testicular tubules of $\mathrm{Pten}^{-1-}$ males (Fig. 6a, bottom panel). Furthermore, we observed the groups of GFR $\alpha 1^{-} / \mathrm{PLZF}^{-} / \mathrm{UTF} 1^{+}$cells located adjacent the cells of GFR $\alpha 1^{+} / \mathrm{PLZF}^{-} / \mathrm{UTF}^{+}{ }^{+}$in the Pten $^{-/-} 7$ dayold testicular tubules (Fig. 6b). Therefore, we hypothesize a model of SSC fate: A GFR $\alpha 1^{+} / \mathrm{PLZF}^{+} / \mathrm{UTF}^{+}$ SSC may undergo asymmetric division, then generate a GFR $\alpha 1^{+} / \mathrm{PLZF}^{+} / \mathrm{UTF}^{+}{ }^{+}$cell for self-renewal and another GFR $\alpha 1^{+} / \mathrm{PLZF}^{-} / \mathrm{UTF}^{+}{ }^{+}$cell. The latter cell enters cell cycle, loses GFR $\alpha 1$ expression, and later develops into differentiating SSCs (Fig. 6c).

\section{Discussion}

Prior to undergoing differentiation of meiosis, spermatogonial stem cells proliferate and form a pool of cells at different division status to meet the dynamics of spermatogenesis. This cell pool is maintained by self-renewal and proliferation of SSCs and exists throughout life time. The transcriptional factor PLZF plays a crucial role in the maintenance of SSC pool in adult males, and PLZF knockout causes a progressive loss of spermatogonia with age $[1,2]$. Interestingly, Pten knockout male pups have less PLZF positive SSCs even at 7 day-old (Figs. 4a, f). We have reported that PLZF expression is regulated by the PTEN signaling pathway in prostate cells [17]. Similarly, PLZF expression was significantly reduced in the Pten knockout SSCs in this study (Fig. 5a), and further experiments in vitro confirmed that in SSCs, PLZF was indeed regulated by the PTEN signaling (Fig. 5b). Thus, in $\mathrm{Pten}^{-1-}$ males, the loss of SSCs is partly due to the reduction of PLZF expression.

The precise regulation of the balance of self-renewal versus differentiation of stem cells is critical in controlling tissue homeostasis and function. Excessive differentiation-associated proliferation leads to depletion of stem cells and degeneration of tissue with age. In $\mathrm{Pten}^{-1-}$ males, although the number of SSCs decreased, which occurred as early as 7 days after birth, testes underwent overgrowth or premature of larger size until day 42 but shrank afterward. This phenotype is apparently associated with excessive differentiation-proliferation of SSCs, which disturbs the long-term maintenance of stem cell pool, thereby leading to the exhaustion of spermatogenesis with age. Furthermore, Pten knockout induced the expression of UTF1, which is expressed in a subpopulation of spermatogonial cells in the testis $[4,16]$. UTF $1^{+}$cells were significantly increased in the testis as early as at day 10 in the Pten $^{-/-}$males compared with wild-type males (Fig. 4d). Using immunostaining of a nearly infertile 32 day-old $\mathrm{Pten}^{-1-}$ testis, many $\mathrm{UTF}^{+}{ }^{+} / \mathrm{PLZF}^{-}$cells and few $\mathrm{UTF}^{-}{ }^{-}$ $\mathrm{PLZF}^{+}$cells (Additional file 1: Figure S1A) were observed, indicating that Pten-deletion-induced UTF1 expression might boost SSCs differentiation associated with the testes overgrowth of Pten knockout males. Moreover, it has been reported that UTF1 makes the spermatogonia maintain the ability of differentiation [4] and is involved in the 


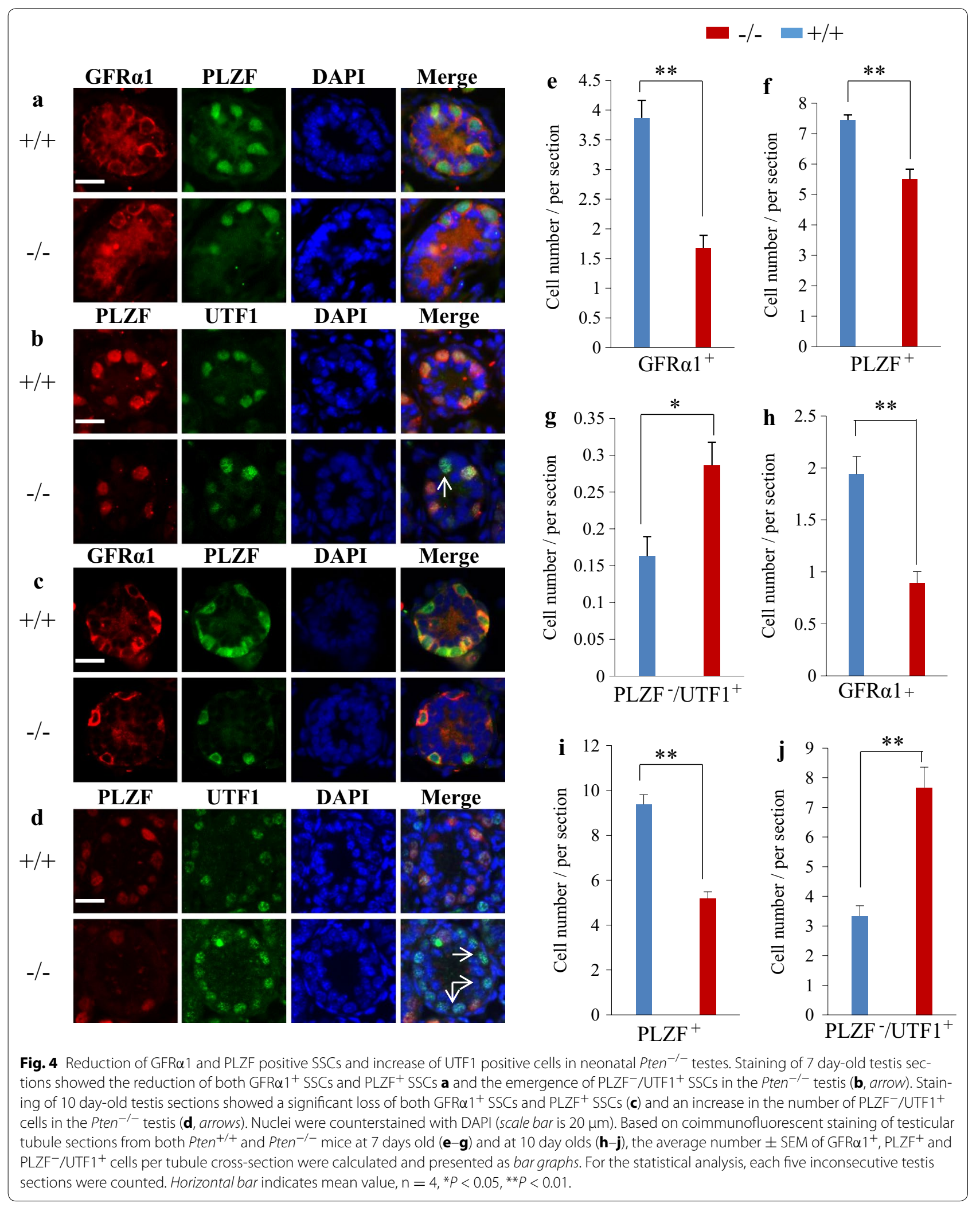




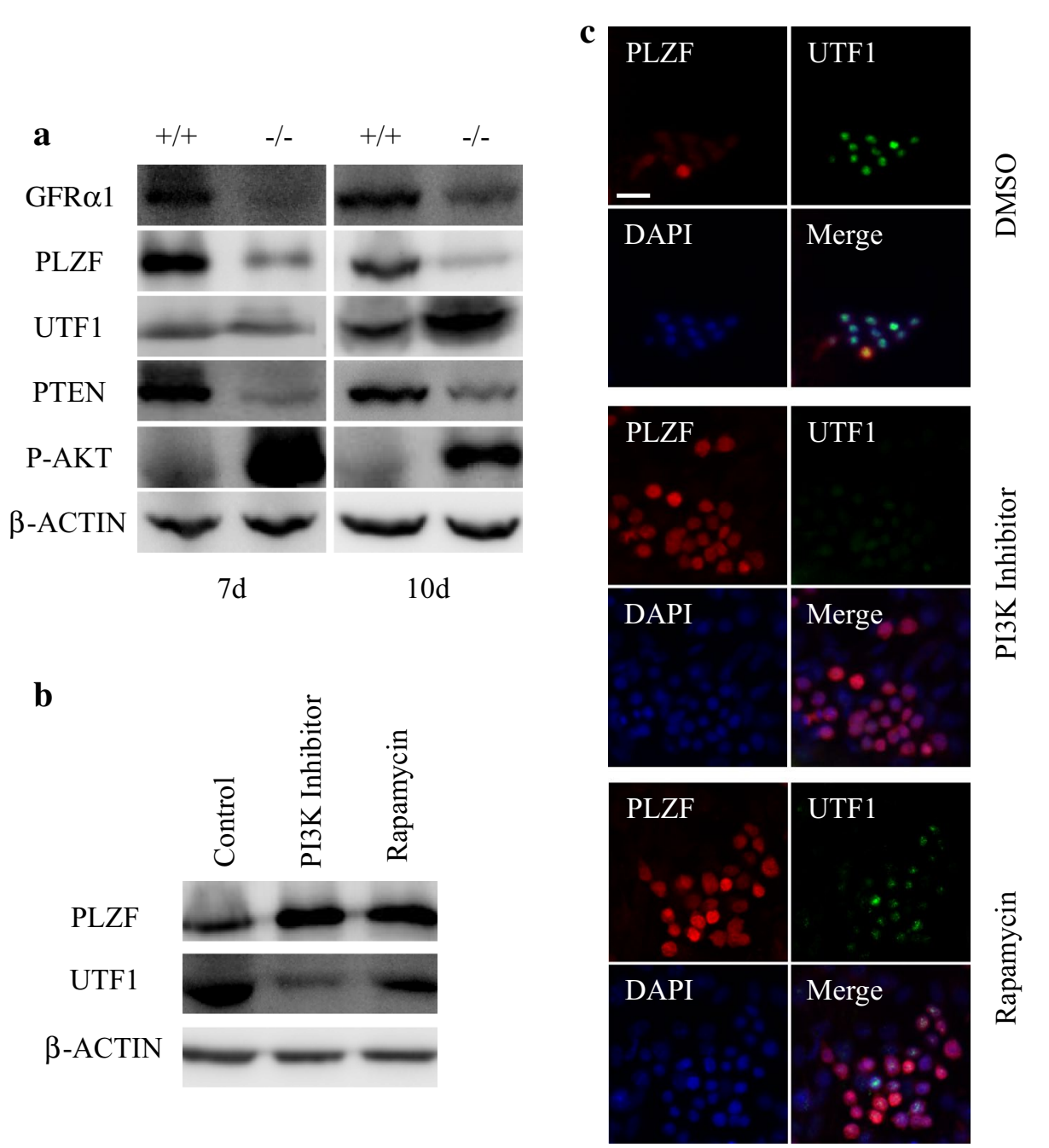

Fig. 5 PLZF and UTF1 are regulated by the PI3K/PTEN signal pathway. a Western blot analysis of GFR 1 1, PLZF, UTF1, PTEN, and P-AKT expression in Pten $^{+/+}$and Pten ${ }^{-/-}$testes at 7 and 10 days old. b Western blot analysis of PLZF and UTF1 expression in cultured SSCs isolated from 7 day-old testes. The treatment with PI3K inhibitor or rapamycin lasted for $96 \mathrm{~h}$, DMSO was used as control. c Coimmunofluorescent staining of PLZF and UTF1 in SSCs treated with PI3K inhibitor, rapamycin or DMSO, showed that the PI3K signal pathway inhibition induced PLZF but reduced UTF1 expression (scale bar is $20 \mu \mathrm{m}$ ).

initiation of ES cell differentiation [15]. All of these results indicate that UTF $1^{+}$cells are differentiating SSCs. To further identify the properties of UTF1 $1^{+}$cells, we performed a three color whole-mount staining of UTF1 with GFR $\alpha 1$ and PLZF of 7 day old tubules to locate UTF1 expression in the SSC population. Notably, a subpopulation of $\mathrm{UTF}^{+} / \mathrm{GFR} \alpha 1^{+} / \mathrm{PLZF}^{-}$SSC and $\mathrm{UTF} 1^{\text {low }} / \mathrm{GFR} \alpha 1^{+} /$ $\mathrm{PLZF}^{+}$cell was observed, which seemed to come from the same precursor cell through asymmetric division. Similar subpopulation cells in Pten $^{-1-}$ tubule lost both GFR $\alpha 1$ and PLZF expression. Therefore, we hypothesized a model of SSC fate in Fig. 6c. Thus, in this study, Pten knockout induced UTF1 expression in addition to causing the loss of PLZF expression. However, further studies should be conducted to address the mechanism how Pten regulates PLZF and UTF1 expression.

Although the testes overgrowth in Pten knockout males occurred within the first 2 months, the fertility and embryos production were lower compared with wild type males at same ages (Fig. 2b). Apparently, this phenotype is associated with the abnormality of sperms found in the epididymis which lack tails (Fig. 3b). This abnormality may be caused by the differentiation defects before haploid stages or during spermatogenesis because Pten is actively expressed in the haploid cells in the testis (Fig. 1d). 


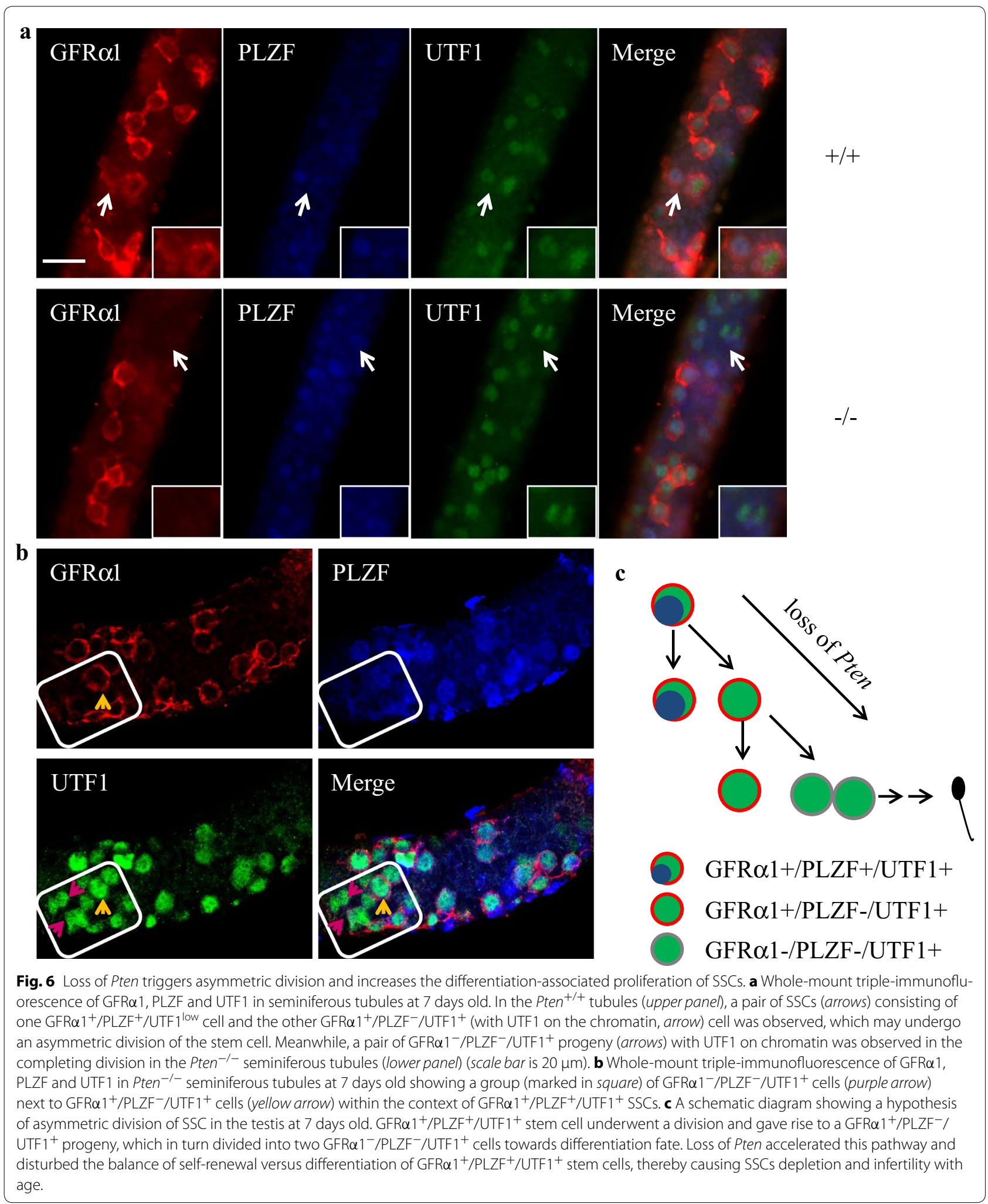


Collectively, The Pten-deletion-induced reduction of PLZF and increased expression of UTF1 apparently disturb the balance of self-renewal and differentiation of SSCs, leading to the depletion of spermatogonial cells and infertility with age.

\section{Conclusion}

By studying the model of Pten knockout in SSCs, we found that Pten is required for the long-term maintenance of SSCs and spermatogenesis. Our study provides a new insight into the precise mechanisms controlling SSC self-renewal versus differentiation to maintain SSC pool and spermatogenesis throughout life time, especially the discovery of a Pten-regulated GFR $\alpha 1^{+} / \mathrm{PLZF}^{-}$/ $\mathrm{UTF} 1^{+}$progenitor population might lead to a new understanding of SSC fate control.

\section{Methods}

\section{Animals}

Stra8-cre mice (Stock number 008208) and Pten ${ }^{\text {LoxP/LoxP }}$ mice (Stock number 006440) were purchased from the Jackson Laboratory. Stra8-cre males were crossed with $\mathrm{Pten}^{\mathrm{f} / \mathrm{f}}$ females to generate Pten knockout in SSCs. Genotype of Stra8-cre mice and Pten ${ }^{\mathrm{f} / \mathrm{f}}$ mice were determined by PCR analysis using the primers and procedures provided by the Jackson Laboratory or by a previous research [18]. For Pten PCR, the Pten ${ }^{\mathrm{f} / \mathrm{f}}(1.1 \mathrm{~kb})$ and Pten $(1 \mathrm{~kb})$ fragments were amplified by using the following primers: $5^{\prime}$-ACTCAAGGCAGGGATGAGC-3' (forward), $5^{\prime}$-AATCTAGGGCCTCTTGTGCC-3' ${ }^{\prime}$ (reverse). For Stra8-cre PCR, the Stra8-cre (179 bp) and Interleukin 2 (Il2 internal positive control, 324 bp) fragments were amplified by using primers: $5^{\prime}$-GTGCAAGCTGA ACAACAGGA-3' (Stra8-cre forward), 5'-AGGGACAC AGCATTGGAGTC-3' (Stra8-cre reverse); 5'-CTAGGCC ACAGAATTGAAAGATCT-3' (Il2 forward), 5'-GTAGG TGGAAATTCTAGCATCATCC-3' (Il2 reverse). Animals used in this study were maintained according to the Guide for the Care and Use of Laboratory Animals (Publication 85-23, revised 1996; National Institutes of Health, Bethesda, MD, USA), and the protocol was approved by Shanghai Jiao Tong University School of Medicine (Shanghai, China)

\section{Histological analysis and immunostaining}

Testes and epididymis were fixed in fresh Bouin's fixative, embedded in paraffin and sectioned at $4 \mu \mathrm{m}$ thickness. After the hematoxylin and eosin staining, the sections were mounted and viewed under a microscope (Carl Zeiss, Maple Grove, MN, USA).

For immunohistochemical staining, testes were fixed in $4 \%$ paraformaldehyde, embedded in OCT and sectioned at $8 \mu \mathrm{m}$ thickness. The endogenous peroxidase activity was blocked by placing the slides in $3 \%$ hydrogen peroxidase for $10 \mathrm{~min}$ followed by a tap water rinse. After being blocked with $5 \%$ BSA, slides were subsequently incubated with the primary antibody against PTEN (1:50 dilution, BOSTER BA1377) at $4^{\circ} \mathrm{C}$ overnight, slides were then incubated with Biotin conjugated secondary antibody. Following incubation with Streptavidin-Biotin Complex (BOSTER SA1022), visualization was performed with a DAB reaction, thereby resulting in brown staining of structures containing the epitope. Cellular nuclei were counterstained with hematoxylin and slides were permanently mounted and evaluated under a light microscope. For immunofluorescent staining, after blocking with $2 \%$ BSA, frozen slides or cell slides were stained with antibodies against PLZF (1:100 dilution, R\&D, AF2944), UTF1 (1:1,000 dilution, ABCAM, ab24273) or GFR $\alpha 1$ (1:40 dilution, R\&D, AF560). The primary antibodies were revealed with Alexa-555 and Alexa-488 conjugated secondary antibodies together with DAPI to stain the nuclei. The sections were mounted and viewed under a fluorescence microscope. For statistical analysis, five different slides from $\mathrm{Pten}^{+/+}$or $\mathrm{Pten}^{-/-}$mice were stained and positive cell numbers were calculated and analyzed by one-way ANOVA $(\alpha=0.05)$. For whole-mount staining, with enzymatic dissociation of the testes using $1 \mathrm{mg} /$ $\mathrm{ml}$ collagenase for $5 \mathrm{~min}$ at $37^{\circ} \mathrm{C}$, untangled seminiferous tubules were fixed with $2 \%$ paraformaldehyde containing $0.5 \mathrm{mM} \mathrm{CaCl}_{2}$ for $30 \mathrm{~min}$ at room temperature. After incubation with $1 \%$ Ttriton 100 for 10 min, samples were dehydrated through a series of methanol $(25,50,75$, and $100 \%$ in PBS containing $0.5 \%$ Triton $100-$ PBS-T) on ice followed by rehydration in PBS-T. The seminiferous tubules were incubated in a blocking buffer $(1 \%$ BSA and $4 \%$ donkey serum) for $1 \mathrm{~h}$ and incubated with the first antibody combination at $4^{\circ} \mathrm{C}$ overnight. The appropriate second antibodies (Alexa-555, Alexa-488 and Dylight-405 conjugated) were applied onto the samples at room temperature for $2 \mathrm{~h}$. After washing with PBS-T, the samples were mounted and observed under a fluorescence microscope.

\section{Western blot analysis}

The proteins were extracted from the cells or testes using the lysis buffer containing $50 \mathrm{mM}$ Tris- $\mathrm{HCl}$ (pH7.4), 1 mM EDTA, $150 \mathrm{mM} \mathrm{NaCl}, 1 \%$ sodium deoxycholate, $0.1 \%$ SDS, $10 \mathrm{mM}$ sodium fluoride, $1 \mathrm{mM}$ sodium orthavanadate and $1 \%$ protease inhibitor cocktail (Sigma-Aldrich Corp, St. Louis, MO, USA). The extracted samples containing $50 \mu \mathrm{g}$ proteins were subjected to $10 \%$ SDS-PAGE and electrophoretically transferred to polyvinylidene difluoride membranes. The filter was probed with PLZF antibody (1:200 dilution, R\&D AF2944), UTF1 antibody (1:250 dilution, Chemicon 
MAB4337), PTEN antibody (1:1,000 dilution, Millipore 04-035), GFR $\alpha 1$ antibody (1:2,000 dilution, R\&D AF560), P-AKT antibody (1:1,000 dilution, Cell Signaling Technology \#4058s) and $\beta$-actin (Cell Signaling Technology). Appropriate secondary antibodies were used and the antibody-antigen complexes in the membranes were visualized using an enhanced-chemiluminescent detection kit (Millipore). The images were scanned using LAS-4000 mini (FUJIFILM, Minato-ku, Tokyo, Japan).

\section{RNA isolation and RT-PCR analysis}

The total RNAs were extracted using TRIzol reagent (Invitrogen) and then the RNAs were reverse transcribed by using a Reverse Transcription kit according to manufacturer's instructions (TaKaRa, DRR037A). The following primers were used for SYBR Green-based real-time PCR (TaKaRa, DRR420A) on a 7900HT Real Time PCR System (Applied Biosystems Inc, USA): Gapdh [GenBank: NM_008084.3], 5'-TGCCCCCATGTTTGTGATG-3' and 5'-TGTGGTCATGAGCCCTTCC-3'; Pten [GenBank: NM_008084.3], 5'-TTCATACCAGGACCAGAGGA-3' and $5^{\prime}$-TTGTCATTATCTGCACGCTCT- $3^{\prime}$. Relative gene expression was calculated by the two DDCt method against internal reference gene of glyceraldehyde-3-phosphate dehydrogenase (Gapdh).

\section{In vivo fertility assay}

To evaluate the effect of $\mathrm{Pten}^{-1-}$ on fertility, we carried out in vivo fertility assay. For each experiment, two normal female mice were mated with one $\mathrm{Pten}^{+/+}$or $\mathrm{Pten}^{-/-}$ male for 2 weeks and then embryos were counted. This mating test was artificially divided into three groups according to the male's ages as follows: 35-60 days, 61-100 days, and older than 100 days. All statistical analyses were conducted with GraphPAD 5.0.

\section{Isolation of haploid cells}

Testes were cut into pieces after removing the tunica albuginea, and testicular fragments in PBS were shocked roughly to wash out the intermediate cells near the lumen. Subsequently, the cells in supernatant were collected and stained with Hoechst $33342(5 \mu \mathrm{g} / \mathrm{ml})$. After 90 min of incubation, cells were resuspended in an icecold cell solution (PBS with 10\% FBS) containing $2 \mu \mathrm{g} /$ $\mathrm{ml}$ of propidium iodide for dead cell discrimination. All the solutions contain verapamil $(50 \mu \mathrm{M} / \mathrm{ml})$ to block the efflux of Hoechst. Finally, sorting was performed on an Influx cell sorter with UV laser (BD Biosciences) [19].

\section{Isolation and culture of spermatogonial stem cells}

Testes were removed from pups with fine forceps using sterile procedures and cut into pieces after removing the tunica albuginea. Following a two-step enzymatic digestion at $37^{\circ} \mathrm{C}$ until the tubules became minimum, supernatants were pipetted and collected quickly. The supernatant was centrifuged to remove the collagenase and the cells were incubated in a dish for $1 \mathrm{~h}$, when the somatic cells had adhered to the bottom of the dish, the supernatants were collected and resuspended in KO-DMEM medium containing 1\% FBS and 1,500 units/ml LIF to 6 well plates (for western blotting) or to 12 well plates with covers in each well (for immunofluorescent staining). Recombinant human GDNF and bFGF were added at a final concentration of 20 and $1 \mathrm{ng} / \mathrm{ml}$ respectively. Cells were maintained at $34^{\circ} \mathrm{C}$ in a humidified $5 \% \mathrm{CO}_{2}$ atmosphere [20]. The medium (containing $5 \mu \mathrm{M}$ PI3K inhibitor or rapamycin $20 \mathrm{nM}$ and growth factors) were changed every other day.

\section{Additional file}

Additional file 1: Figure S1. (A) Coimmunofluorescent staining of PLZF and UTF1 in 32 day-old Pten $^{-1-}$ cross sections showing a number of $\mathrm{UTF}^{+} / \mathrm{PLZF}^{-}$cells and few $\mathrm{UTF} 1^{-} / \mathrm{PLZF}^{+}$cells. (scale bar is $50 \mu \mathrm{m}$ ). (B) Whole transcriptome sequencing result of 32 day old $P$ ten $^{+/+}$and Pten ${ }^{-/-}$ testes showing that UTF1 expression was significantly increased in the Pten $^{-1-}$ testes.

\section{Abbreviations}

PTEN: phosphatase and tensin homolog; PLZF: promyelocytic leukaemia zinc finger; UTF1: undifferentiated embryonic cell transcription factor 1; PI(3) K: phosphatidylinositol-3-OH kinase; SSCs: spermatogonial stem cells; Stra8: stimulated by retinoic acid gene 8; EGFP: enhanced green fluorescent protein; GFR 1: GDNF family receptor alpha-1; FOXO3A: forkhead box O3; II2: interleukin 2; LIF: leukemia inhibitory factor; FBS: fetal bovine serum; GDNF: glial cell-derived neurotrophic factor; bFGF: basic fibroblast growth factor.

\section{Authors' contributions}

DZ, JD helped with the mice manipulation, WC and LW did the SSC isolation and culture experiments, WZ performed most of the experiments, HS and YT prepared the manuscript, $Z Y$ and $W Z$ designed the research and prepared the manuscript. All authors read and approved the final manuscript.

\section{Author details}

${ }^{1}$ Hongqiao International Institute of Medicine, Shanghai Tongren Hospital / Faculty of Basic Medicine, Shanghai Jiao Tong University School of Medicine, Shanghai 200025, China. ${ }^{2}$ Key Laboratory of Cell Differentiation and Apoptosis of Chinese Ministry of Education, Shanghai Jiao Tong University School of Medicine, Shanghai 200025, China. ${ }^{3}$ Centre for Reproductive Medicine, Shanghai Jiao Tong University Affiliated Sixth People Hospital, Shanghai 200233, China. ${ }^{4}$ Institute of Genetics and Developmental Biology, Chinese Academy of Sciences, Beijing 100101, China. ${ }^{5}$ College of Public Health, Shanghai Jiao Tong University School of Medicine, Shanghai 200025, China.

\section{Acknowledgements}

The other authors wish to dedicate this paper to Dr. Lixin Feng who passed away during the manuscript preparation. This study was supported by Grants from the National Natural Science Foundation of China (31171409, 81000049, 81370654), National Key Basic Research and Development Program of China (2012CB966603), Innovation Program of Shanghai Municipal Education Commission (13G20), and the Program for Professor of Special Appointment (Eastern Scholar) at Shanghai Institutions of Higher Learning.

\section{Compliance with ethical guidelines}

\section{Competing interests}

The authors declare that they have no competing interests. 
Received: 21 April 2015 Accepted: 11 July 2015

Published online: 28 July 2015

\section{References}

1. Buaas FW, Kirsh AL, Sharma M, McLean DJ, Morris JL, Griswold MD, et al. Plzf is required in adult male germ cells for stem cell self-renewal. Nat Genet. 2004;36(6):647-52. doi:10.1038/ng1366ng1366.

2. Costoya JA, Hobbs RM, Barna M, Cattoretti G, Manova K, Sukhwani M, et al. Essential role of Plzf in maintenance of spermatogonial stem cells. Nat Genet. 2004;36(6):653-9. doi:10.1038/ng1367ng1367.

3. He Z, Jiang J, Hofmann MC, Dym M. Gfra1 silencing in mouse spermatogonial stem cells results in their differentiation via the inactivation of RET tyrosine kinase. Biol Reprod. 2007;77(4):723-33. doi:10.1095/ biolreprod.107.062513.

4. van Bragt MP, Roepers-Gajadien HL, Korver CM, Bogerd J, Okuda A, Eggen BJ, et al. Expression of the pluripotency marker UTF1 is restricted to a subpopulation of early A spermatogonia in rat testis. Reproduction. 2008;136(1):33-40. doi:10.1530/REP-07-0536.

5. Jung H, Roser JF, Yoon M. UTF1, a putative marker for spermatogonial stem cells in stallions. PLoS One. 2014;9(10):e108825. doi:10.1371/journal. pone.0108825.

6. Groszer M, Erickson R, Scripture-Adams DD, Lesche R, Trumpp A, Zack JA et al. Negative regulation of neural stem/progenitor cell proliferation by the Pten tumor suppressor gene in vivo. Science. 2001;294(5549):2186-9. doi:10.1126/science.1065518.

7. Zhang J, Grindley JC, Yin T, Jayasinghe S, He XC, Ross JT, et al. PTEN maintains haematopoietic stem cells and acts in lineage choice and leukaemia prevention. Nature. 2006;441(7092):518-22. doi:10.1038/nature04747.

8. Reddy P, Liu L, Adhikari D, Jagarlamudi K, Rajareddy S, Shen Y, et al. Oocyte-specific deletion of Pten causes premature activation of the primordial follicle pool. Science. 2008;319(5863):611-3. doi:10.1126/ science.1152257.

9. Blume-Jensen P, Jiang G, Hyman R, Lee KF, O'Gorman S, Hunter T. Kit/ stem cell factor receptor-induced activation of phosphatidylinositol 3'-kinase is essential for male fertility. Nat Genet. 2000;24(2):157-62. doi:10.1038/72814.
10. Feng LX, Ravindranath N, Dym M. Stem cell factor/c-kit up-regulates cyclin D3 and promotes cell cycle progression via the phosphoinositide 3-kinase/p70 S6 kinase pathway in spermatogonia. J Biol Chem. 2000;275(33):25572-6. doi:10.1074/jbc.M002218200.

11. Maehama T, Dixon JE. The tumor suppressor, PTEN/MMAC1, dephosphorylates the lipid second messenger, phosphatidylinositol 3,4,5-trisphosphate. J Biol Chem. 1998;273(22):13375-8.

12. Stambolic V, Suzuki A, de la Pompa JL, Brothers GM, Mirtsos C, Sasaki $\mathrm{T}$, et al. Negative regulation of PKB/Akt-dependent cell survival by the tumor suppressor PTEN. Cell. 1998;95(1):29-39.

13. Oulad-Abdelghani M, Bouillet P, Decimo D, Gansmuller A, Heyberger S, Dolle $P$, et al. Characterization of a premeiotic germ cell-specific cytoplasmic protein encoded by Stra8, a novel retinoic acid-responsive gene. J Cell Biol. 1996;135(2):469-77.

14. Giuili G, Tomljenovic A, Labrecque N, Oulad-Abdelghani M, Rassoulzadegan M, Cuzin F. Murine spermatogonial stem cells: targeted transgene expression and purification in an active state. EMBO Rep. 2002;3(8):753-9. doi:10.1093/embo-reports/kvf149.

15. van den Boom V, Kooistra SM, Boesjes M, Geverts B, Houtsmuller AB, Monzen K, et al. UTF1 is a chromatin-associated protein involved in ES cell differentiation. J Cell Biol. 2007;178(6):913-24. doi:10.1083/jcb.200702058.

16. Kristensen DM, Nielsen JE, Skakkebaek NE, Graem N, Jacobsen GK, Rajpert-De Meyts E, et al. Presumed pluripotency markers UTF-1 and REX-1 are expressed in human adult testes and germ cell neoplasms. Hum Reprod. 2008;23(4):775-82. doi:10.1093/humrep/den010.

17. Cao J, Zhu S, Zhou W, Li J, Liu C, Xuan H, et al. PLZF mediates the PTEN/ AKT/FOXO3a signaling in suppression of prostate tumorigenesis. PLOS One. 2013;8(12):e77922. doi:10.1371/journal.pone.0077922.

18. Ruan H, Li J, Ren S, Gao J, Li G, Kim R, et al. Inducible and cardiac specific PTEN inactivation protects ischemia/reperfusion injury. J Mol Cell Cardiol. 2009;46(2):193-200. doi:10.1016/j.yjmcc.2008.10.021.

19. Goodell MA. Stem cell identification and sorting using the Hoechst 33342 side population (SP). In: Paul Robinson J et al., editors Current protocols in cytometry/editorial board, Chapter 9:Unit9.18. 2005. doi:10.1002/0471142956.cy0918s34.

20. Wang L, Cao J, Ji P, Zhang D, Ma L, Dym M, et al. Oocyte-like cells induced from mouse spermatogonial stem cells. Cell Biosci. 2012;2(1):27. doi:10.1186/2045-3701-2-27

\section{Submit your next manuscript to BioMed Central and take full advantage of:}

- Convenient online submission

- Thorough peer review

- No space constraints or color figure charges

- Immediate publication on acceptance

- Inclusion in PubMed, CAS, Scopus and Google Scholar

- Research which is freely available for redistribution

Submit your manuscript at

www.biomedcentral.com/submit

C BioMed Central 